\title{
Dimension reduction for compressible Navier-Stokes equations with density-dependent viscosity
}

Mingyu Zhang ${ }^{1 *}$ (i)

\section{"Correspondence:}

myuzhang@126.com

${ }^{1}$ School of Mathematics \&

Information Sciences, Weifang

University, Weifang, P.R. China

\section{Springer}

\begin{abstract}
In this paper, we investigate the Navier-Stokes equations describing the motion of a compressible viscous fluid confined to a thin domain $\Omega_{\varepsilon}=I_{\varepsilon} \times(0,1), I_{\varepsilon}=(0, \varepsilon) \subset \mathbb{R}$. We show that the strong solutions in the $2 \mathrm{D}$ domain converge to the classical solutions of the limit 1D Navier-Stokes system as $\varepsilon \rightarrow 0$.
\end{abstract}

MSC: $35 \mathrm{Q} 35 ; 76 \mathrm{D} 05 ; 76 \mathrm{~N} 10$

Keywords: Compressible Navier-Stokes equations; Dimension reduction; Relative entropy

\section{Introduction}

We consider the Navier-Stokes system for a barotropic compressible viscous fluid which in the case of two-dimensional motion has the form $[10,15,20]$

$$
\left\{\begin{array}{l}
\partial_{t} \rho_{\varepsilon}+\operatorname{div}_{x}\left(\rho_{\varepsilon} u_{\varepsilon}\right)=0 \\
\partial_{t}\left(\rho_{\varepsilon} u_{\varepsilon}\right)+\operatorname{div}_{x}\left(\rho_{\varepsilon} u_{\varepsilon} \otimes u_{\varepsilon}\right)+\nabla_{x} P\left(\rho_{\varepsilon}\right)=\operatorname{div}_{x} \mathbb{S}\left(\nabla_{x} u_{\varepsilon}\right),
\end{array}\right.
$$

where $\rho_{\varepsilon}=\rho_{\varepsilon}(x, t)$ and $u_{\varepsilon}=\left(u_{\varepsilon}^{1}(x, t), u_{\varepsilon}^{2}(x, t)\right)$ stand for the unknown fluid mass density and the velocity field, respectively, and the viscous stress tensor $\mathbb{S}$ is a linear function of the velocity gradient and therefore described by the Newton law:

$$
\mathbb{S}\left(\nabla_{x} u_{\varepsilon}\right)=\mu\left(\nabla_{x} u_{\varepsilon}+\nabla_{x}^{t} u_{\varepsilon}\right)+\lambda\left(\rho_{\varepsilon}\right) \operatorname{div}_{x} u_{\varepsilon} \mathbb{I},
$$

the shear viscosity coefficient $\mu$, the bulk one $\lambda$ and the pressure $P$ are defined on $(0,+\infty)$ and satisfy the conditions

$$
0<\mu=\text { const }, \quad \lambda\left(\rho_{\varepsilon}\right)=b \rho_{\varepsilon}^{\beta}, \quad P\left(\rho_{\varepsilon}\right)=a \rho_{\varepsilon}^{\gamma}
$$

(c) The Author(s) 2020. This article is licensed under a Creative Commons Attribution 4.0 International License, which permits use, sharing, adaptation, distribution and reproduction in any medium or format, as long as you give appropriate credit to the original author(s) and the source, provide a link to the Creative Commons licence, and indicate if changes were made. The images or other third party material in this article are included in the article's Creative Commons licence, unless indicated otherwise in a credit line to the material. If material is not included in the article's Creative Commons licence and your intended use is not permitted by statutory regulation or exceeds the permitted use, you will need to obtain permission directly from the copyright holder. To view a copy of this licence, visit http://creativecommons.org/licenses/by/4.0/. 
for positive constants $a>0, b>0$. In the sequel, we set $a=b=1$ without loss of generality, and we also have the following hypotheses on $\gamma$ and $\beta$ :

$$
\beta>3, \quad \gamma>1 .
$$

The fluid is confined to a bounded physical domain $\Omega_{\varepsilon} \subset \mathbb{R}^{2}$, on the boundary of which we impose the complete slip boundary conditions

$$
\left.u_{\varepsilon} \cdot n\right|_{\partial \Omega_{\varepsilon}}=0, \quad\left[\mathbb{S}\left(\nabla_{x} u_{\varepsilon}\right) \cdot n\right] \times\left. n\right|_{\partial \Omega_{\varepsilon}}=0,
$$

where the symbol $n$ denotes the outer normal vector. The motion originates from the initial state

$$
\rho_{\varepsilon}(\cdot, 0)=\rho_{0, \varepsilon}, \quad u_{\varepsilon}(\cdot, 0)=u_{0, \varepsilon}, \quad x \in \Omega_{\varepsilon} .
$$

We remark that the use of the slip instead of the more conventional no-slip condition $\left.u_{\varepsilon}\right|_{\partial \Omega_{\varepsilon}}=0$ is quite natural in the present context as the latter would completely stop the fluid motion in the asymptotic limit $\varepsilon \rightarrow 0$.

Although all fluid flows are in general two-dimensional, in many cases the specific shape of the physical domain enforce major changes in the density and velocity only in one direction. A typical example is the fluid flow confined to a thin domain that can be effectively described by using only spatial variable. We consider a family of shrinking domains:

$$
\Omega_{\varepsilon}=I_{\varepsilon} \times(0,1), \quad I_{\varepsilon} \subset \mathbb{R}, \quad I_{\varepsilon}=\varepsilon I \subset \mathbb{R}, \quad \varepsilon \rightarrow 0,
$$

where $I \subset \mathbb{R}$ is a unit interval. Under suitable conditions on the initial data it is natural to expect that the strong solution $\left(\rho_{\varepsilon}, u_{\varepsilon}\right)$ of $(1.1)-(1.2)$ on $\Omega_{\varepsilon}$ tends, as $\varepsilon \rightarrow 0$, to a classical solution $(\rho, u)$ of the $1 \mathrm{D}$ system on $(0,1)$ :

$$
\left\{\begin{array}{l}
\partial_{t} \rho+\partial_{y}(\rho u)=0, \\
\partial_{t}(\rho u)+\partial_{y}\left(\rho u^{2}+P(\rho)\right)=\partial_{y}\left(v(\rho) \partial_{y} u\right), \quad v(\rho)=2 \mu+\lambda(\rho) .
\end{array}\right.
$$

The boundary conditions (1.2) naturally lead to the no-slip boundary conditions for the velocity, i.e.,

$$
u(0, \cdot)=u(1, \cdot)=0
$$

Since we are interested in smooth solutions of the 1D equations, we complement the system of equations (1.4) and (1.5) with the initial conditions

$$
\rho(\cdot, 0)=\rho_{0}, \quad u(\cdot, 0)=u_{0}, \quad x \in(0,1) .
$$

Hereinafter we use the notation $x=\left(x_{1}, y\right) \in \mathbb{R}^{2}, y \in \mathbb{R}$ and denote the derivative in $x_{2}$ by $\partial_{y}$. In this paper we give a rigorous justification of the convergence $\left(\rho_{\varepsilon}, u_{\varepsilon}\right) \rightarrow(\rho, u)$ as $\varepsilon \rightarrow 0$. As far as we know, the limit passage for fluid flows has not yet been rigorously investigated and there is only a handful of results on related problems. Since incompressibility 
in one dimension does not allow for any movement, such a limit makes little sense for 1D incompressibility flows. However, dimension reduction to 2D-planar flows was examined in [11, 17-19]; see also the references given therein.

The case of a compressible barotropic fluid was studied by many authors. Vodák in [22] studied the steady and nonsteady Navier-Stokes system for barotropic compressible flow. For three-dimensional system, Bella, Feireisl and Novotný in [1] considered the motion of a compressible viscous fluid confined to a cavity shaped as a thin $\operatorname{rod} \Omega_{\varepsilon}=\varepsilon Q \times(0,1), Q \subset$ $\mathbb{R}^{2}$, they showed that the weak solutions in the 3D domain converge to (strong) solutions of the limit 1D Navier-Stokes system as $\varepsilon \rightarrow 0$. Březina-Kreml-Mácha in [2] studied the dimension reduction for the full Navier-Stokes-Fourier system in a thin long pipe $\Omega_{\varepsilon}=$ $\varepsilon Q \times(0,1) \in \mathbb{R}^{3}$, where $Q$ is an open rectangular domain in $\mathbb{R}^{2}$, they showed that the weak solutions of 3D system on $\Omega_{\varepsilon}$ tend, as $\varepsilon \rightarrow 0$, to a classical solution of 1D system on $(0,1)$. Ducomet-Caggio-Nečasová-Pokorný in [5] investigated the rotating NavierStokes-Fourier-Poisson system confined to a straight layer $\Omega_{\varepsilon}=\omega \times(0, \varepsilon)$, where $\omega$ is a 2 -D domain, they showed that the weak solutions in the $3 \mathrm{~d}$ domain converge to the strong solution of the 2-D system on $\omega \rightarrow 0$ as $\varepsilon \rightarrow 0$ on the time interval, where the strong solution exists.

Motivated by [1, 5] and [2], our main purpose in this paper is to show that the strong solution of 2D compressible Navier-Stokes system confined to a thin domain $\Omega_{\varepsilon}=(0, \epsilon) \times$ $(0,1)$ converge to the classical solution of the 1D Navier-Stokes system on $(0,1)$ as $\varepsilon \rightarrow 0$.

In elasticity theory, the analysis of similar dimension reduction problems leans on variants of the Korn inequality which controls the gradient of velocity $v$ by its symmetric part, specifically,

$$
\left\|\nabla_{x} v\right\|_{L^{2}\left(\Omega_{\varepsilon}\right)} \leq c(\varepsilon)\left\|\nabla_{x} v+\nabla_{x}^{t} v\right\|_{L^{2}\left(\Omega_{\varepsilon}\right)},\left.\quad v \cdot n\right|_{\partial \Omega_{\varepsilon}}=0 .
$$

Two problems have arisen in (1.7). Firstly, the kernel of the linear operator $v \mapsto \nabla_{x} v+$ $\nabla_{x}^{t} v,\left.v \cdot n\right|_{\partial \Omega_{\varepsilon}}$ has to be empty, in particular, the "bottom" set $I$ must not be rotationally symmetric. Secondly, for any fixed $\varepsilon>0$, even if (1.7) holds, the constant $c(\varepsilon)$ blows up for $\varepsilon \rightarrow 0$ unless some necessary restrictions are imposed on the field $v$, and this is true even if the set $I$ is not rotationally symmetric, cf. the interesting paper by Lewicka and Müller in [14].

Bella, Feireisl and Novotný in [1] obtained their result for a regular planar domain since they avoid the use of Kron's inequality by exploring the structural stability of the family of solutions of the barotropic Navier-Stokes system. It is not difficult to see that the problems arising in the context of compressible fluids would need a stronger analogue of (1.7), namely

$$
\left\|\nabla_{x} v\right\|_{L^{2}\left(\Omega_{\varepsilon}\right)} \leq c(\varepsilon)\left\|\nabla_{x} v+\nabla_{x}^{t} \nu-\frac{2}{3} \operatorname{div}_{x} v \mathbb{I}\right\|_{L^{2}\left(\Omega_{\varepsilon}\right)},\left.\quad v \cdot n\right|_{\partial \Omega_{\varepsilon}}=0 .
$$

In view of the above-mentioned difficulties related to the validity of (1.7) or (1.8), our approach relies on the structural stability of the family of solutions of the barotropic NavierStokes system encoded in the relative entropy inequality introduced in $[6,8]$. This method is basically independent of the specific form of the viscous stress and of possible "dissipative" bounds for the Navier-Stokes system. 
The paper is organized as follows. In Sect. 2 we introduce the relative entropy inequality and formulate our main result. In Sect. 3, we establish convergence towards the target system (1.4).

\section{Preliminaries, main result}

In this section, we first introduce the relative entropy inequality, and then give the solutions of the target systems (1.1) and (1.4), finally, we state our main result.

\subsection{Relative entropy inequality}

The proof of our main theorem is based on the method of the relative entropy (see [8], Dafermos in [3], Germain in [9] and Mellet, Vasseur in [16]). The relative entropy $\mathcal{E}_{\varepsilon}([\rho, u] \mid[r, U])$ with respect to $[r, U]$ is defined as

$$
\mathcal{E}_{\varepsilon}([\rho, u] \mid[r, U])=\frac{1}{\left|I_{\varepsilon}\right|} \int_{0}^{1} \int_{I_{\varepsilon}}\left(\frac{1}{2} \rho|u-U|^{2}+H(\rho)-H^{\prime}(r)(\rho-r)-H(r)\right) d x_{1} d x_{2}
$$

where the potential $H(\rho)$ is defined (modulo a linear function) through

$$
H(\rho)=\rho \int_{\tilde{\rho}}^{\rho} \frac{P(s)}{s^{2}} d s
$$

then

$$
H^{\prime}(\rho) \rho-H(\rho)=P(\rho)
$$

along with the relative entropy inequality

$$
\begin{aligned}
& \mathcal{E}_{\varepsilon}([\rho, u] \mid[r, U])(t)+\frac{1}{\left|I_{\varepsilon}\right|} \int_{0}^{\tau} \int_{\Omega_{\varepsilon}} \mathbb{S}\left(\nabla_{x}(u-U)\right): \nabla_{x}(u-U) d x d t \\
& \quad \leq \mathcal{E}_{\varepsilon}([\rho, u] \mid[r, U])(0)+\int_{0}^{\tau} \mathcal{F}_{\varepsilon}(\rho, u, r, U) d t
\end{aligned}
$$

and the remainder $\mathcal{F}_{\varepsilon}$ reads

$$
\begin{aligned}
\mathcal{F}_{\varepsilon}(\rho, u, r, U)= & \frac{1}{\left|I_{\varepsilon}\right|} \int_{\Omega_{\varepsilon}} \rho\left(U_{t}+u \cdot \nabla_{x} U\right) \cdot(U-u) d x \\
& +\frac{1}{\left|I_{\varepsilon}\right|} \int_{\Omega_{\varepsilon}} r\left(U_{t}+U \cdot \nabla_{x} U\right) \cdot(u-U) d x \\
& +\frac{1}{\left|I_{\varepsilon}\right|} \int_{\Omega_{\varepsilon}}\left[P(r)-P(\rho)+(\rho-r) P^{\prime}(r)\right] \operatorname{div}_{x} U d x \\
& +\frac{1}{\left|I_{\varepsilon}\right|} \int_{\Omega_{\varepsilon}}(\rho-r)(u-U) \cdot \nabla_{x} H^{\prime}(r) d x .
\end{aligned}
$$

Here, the functions $r, U$ are arbitrary smooth, $r$ strictly positive, and $U$ satisfying the noslip boundary conditions (1.5). It is easy to check that (2.3) is satisfied as an equality as soon as solution $\rho, u$ is smooth enough. 


\subsection{Solutions of target systems (1.1) and (1.4)}

The existence of global-in-time strong solutions to the two-dimensional Navier-Stokes system (1.1) with complete slip boundary conditions was established by Vaigant and Kazhikhov in [21]. It reads as follows.

Proposition 2.1 Let $\Omega \subset \mathbb{R}^{2}$ be a rectangular domain. Assume that

$$
0<\mu=\text { const }, \quad \lambda(\rho)=\rho^{\beta}, \quad P(\rho)=\rho^{\gamma} \quad \text { for } \beta>3, \gamma>1 .
$$

If the initial data $\left(\rho_{0}, u_{0}\right)$ are such that

$$
\left\{\begin{array}{l}
\rho_{0}(x) \in W^{1, q}(\Omega), \quad u_{0}(x) \in H^{2}(\Omega), \quad q>2, \\
0<m_{0} \leq \rho_{0} \leq M_{0}<+\infty
\end{array}\right.
$$

where $m_{0}$ and $M_{0}$ are some positive constants, and the agreement conditions

$$
\left\{\begin{array}{l}
u_{0}^{1}=\partial_{1} u_{0}^{2}=0 \quad \text { on }\left\{x \in \Omega \mid x_{1}=0, \varepsilon\right\} \\
u_{0}^{2}=\partial_{2} u_{0}^{1}=0 \quad \text { on }\left\{x \in \Omega \mid x_{2}=0,1\right\} \\
{\left.\left[\partial_{1}\left(2 \mu \partial_{1} u_{0}^{1}\right)+\partial_{1}\left(\lambda\left(\rho_{0}\right)\left(\partial_{1} u_{0}^{1}+\partial_{2} u_{0}^{2}\right)\right)+\partial_{2}\left(\mu\left(\partial_{2} u_{0}^{2}+\partial_{1} u_{0}^{2}\right)\right)-\partial_{1}\left(P\left(\rho_{0}\right)\right)\right]\right|_{x_{1}=0, \varepsilon}=0} \\
{\left.\left[\partial_{2}\left(2 \mu \partial_{2} u_{0}^{2}\right)+\partial_{2}\left(\lambda\left(\rho_{0}\right)\left(\partial_{1} u_{0}^{1}+\partial_{2} u_{0}^{2}\right)\right)+\partial_{1}\left(\mu\left(\partial_{2} u_{0}^{2}+\partial_{1} u_{0}^{2}\right)\right)-\partial_{2}\left(P\left(\rho_{0}\right)\right)\right]\right|_{x_{2}=0,1}=0}
\end{array}\right.
$$

are satisfied for all $x \in \bar{\Omega}$, where $\partial_{i}$ denotes $\partial_{x_{i}}, i=1,2$, then there exists a unique global strong solution $(\rho, u)$ to problem $(1.1)-(1.2)$ in $\Omega \times(0, \infty)$ satisfying

$$
\left\{\begin{array}{l}
\rho \in L^{q}\left(0, T ; W^{1, q}(\Omega)\right), \quad \partial_{t} \rho \in L^{\infty}\left(0, T ; L^{\infty}(\Omega)\right), \\
u \in L^{2}\left(0, T ; H^{2}(\Omega)\right), \quad \partial_{t} u \in L^{2}\left(0, T ; L^{2}(\Omega)\right),
\end{array}\right.
$$

for any $0<T<\infty$.

Remark 2.1 From [1, 21] and [6], we know that, for two-dimensional compressible barotropic Navier-Stokes system, the global-in-time solution $\left(\rho_{\varepsilon}, u_{\varepsilon}\right)$ of Eqs. (1.1) enjoys the regularity specified in Proposition 2.1, the relative entropy inequality in (2.3) is satisfied for $\rho=\rho_{\varepsilon}, u=u_{\varepsilon}$ and any pair of the test functions

$$
r \in C^{\infty}\left([0, T] \times \bar{\Omega}_{\varepsilon}\right), \quad r>0, \quad U \in C^{\infty}\left([0, T] \times \bar{\Omega}_{\varepsilon}\right),\left.\quad U \cdot n\right|_{\partial \Omega_{\varepsilon}}=0,
$$

and by means of density arguments, the class of test functions can be extended to less regular $(r, U)$.

For one-dimensional compressible Navier-Stokes system (1.4)-(1.6) with no-slip boundary conditions, it has been discussed by many mathematicians. Kazhikhov and Shelukhi in [13] (for polytropic perfect gas with constant viscosity) and Kawohl in [12] (for real gas with $\mu=\mu(\rho))$ got global classical solutions for large initial data with inf $\rho_{0}>0$, respectively. Ding, Wen and Zhu in [4] obtained the global existence of classical solutions to the compressible Navier-Stokes equations in 1D when the initial data are large and the density dependent viscosity. For completeness, we state the proposition as follows. 
Proposition 2.2 Let $\Omega \in \mathbb{R}$ be an unit interval. Assume that (2.5) holds, and the initial data $\left(\rho_{0}, u_{0}\right)$ satisfying

$$
\inf \rho_{0}>0, \quad \rho_{0} \in H^{2}(\Omega), \quad u_{0} \in H^{3}(\Omega) \cap H_{0}^{1}(\Omega)
$$

and the agreement condition

$$
\partial_{y}\left[v\left(\rho_{0}\right) \partial_{y} u_{0}\right](y)-\partial_{y}\left[P\left(\rho_{0}\right)\right](y)=\rho_{0}(y) g(y), \quad y \in[0,1],
$$

for a given function $g \in L^{2}(\Omega)$. Then, for any $T>0$, there exists a unique global classical solution $(\rho, u)$ to $(1.4)-(1.6)$ satisfying

$$
\begin{aligned}
& \left(\rho, \rho^{\gamma}\right) \in C\left([0, T] ; H^{2}(\Omega)\right), \quad\left(\rho_{t},\left(\rho^{\gamma}\right)_{t}\right) \in C\left([0, T] ; H^{1}(\Omega)\right), \\
& \rho_{t t} \in C\left([0, T] ; L^{2}(\Omega)\right), \quad\left(\rho^{\gamma}\right)_{t t} \in L^{\infty}\left([0, T] ; L^{2}(\Omega)\right), \quad(\rho u)_{t} \in C\left([0, T] ; H^{1}(\Omega)\right), \\
& u \in C\left([0, T] ; H^{3}(\Omega) \cap H_{0}^{1}(\Omega)\right), \quad u_{t} \in L^{\infty}\left([0, T] ; H_{0}^{1}(\Omega)\right) \cap L^{2}\left([0, T] ; H^{2}(\Omega)\right) .
\end{aligned}
$$

\subsection{Main result}

We are ready to state our main result.

Theorem 2.1 Let $I_{\varepsilon}=(0, \varepsilon) \subset \mathbb{R}$ and $\Omega_{\varepsilon}=I_{\varepsilon} \times(0,1)$ for $\varepsilon>0$. Suppose that the system (1.1)-(1.2) admits a strong solution $\left(\rho_{\varepsilon}, u_{\varepsilon}\right)$ in $\Omega_{\varepsilon} \times(0, T)$ which emanating from the initial data $\left(\rho_{0, \varepsilon}, u_{0, \varepsilon}\right)$, and the system (1.4)-(1.6) possesses a classical solution $(\rho, u)$ in $(0,1) \times$ $(0, T)$ emanating from $\left(\rho_{0}, u_{0}\right)$.

Let

$$
\frac{1}{\left|I_{\varepsilon}\right|} \int_{I_{\varepsilon}} \rho_{0, \varepsilon}\left(x_{1}, \cdot\right) d x_{1} \rightarrow \rho_{0}, \quad \frac{1}{\left|I_{\varepsilon}\right|} \int_{I_{\varepsilon}} \rho_{0, \varepsilon} u_{0, \varepsilon}\left(x_{1}, \cdot\right) d x_{1} \rightarrow \rho_{0} v_{0}
$$

weakly in $L^{1}(0,1)$, where inf $\rho_{0}>0, v_{0}=\left(0, u_{0}\right)$, and let

$$
\frac{1}{\left|I_{\varepsilon}\right|} \int_{\Omega_{\varepsilon}}\left[\frac{1}{2} \rho_{0, \varepsilon}\left|u_{0, \varepsilon}\right|^{2}+H\left(\rho_{0, \varepsilon}\right)\right] d x \rightarrow \int_{0}^{1}\left[\frac{1}{2} \rho_{0}\left|v_{0}\right|^{2}+H\left(\rho_{0}\right)\right] d y
$$

where $H(\cdot)$ defined as in (2.2). Then

$$
\begin{aligned}
& \text { ess } \sup _{t \in(0, T)} \frac{1}{\left|I_{\varepsilon}\right|}\left\|\rho_{\varepsilon}-\rho\right\|_{L^{\gamma}\left(\Omega_{\varepsilon}\right)}^{\gamma} \rightarrow 0, \\
& \frac{1}{\left|I_{\varepsilon}\right|}\left\|u_{\varepsilon}^{2}-u\right\|_{L^{2}\left(\Omega_{\varepsilon} \times(0, T)\right)}^{2} \rightarrow 0,
\end{aligned}
$$

as $\varepsilon \rightarrow 0$, where $u_{\varepsilon}=\left(u_{\varepsilon}^{1}, u_{\varepsilon}^{2}\right)$.

\section{Proof of Theorem 2.1}

Let $\underline{\rho}, \bar{\rho}>0$ be two positive constants such that

$$
0<\underline{\rho} \leq \frac{1}{2} \inf _{(y, t) \in(0,1) \times(0, T)} \rho(y, t) \leq 2 \sup _{(y, t) \in(0,1) \times(0, T)} \rho(y, t) \leq \bar{\rho} .
$$


Now, we introduce the set of essential values $\mathcal{O}_{\text {ess }} \subset(0, \infty)$

$$
\mathcal{O}_{\text {ess }} \triangleq\left\{\rho_{\epsilon} \in \mathbb{R} \mid \underline{\rho} \leq \rho_{\varepsilon} \leq \bar{\rho}\right\},
$$

together with its residual counterpart

$$
\mathcal{O}_{\text {res }} \triangleq(0, \infty) \backslash \mathcal{O}_{\text {ess }} .
$$

Then each measurable function $h$ can be written as

$$
h=h_{\mathrm{ess}}+h_{\mathrm{res}},
$$

where

$$
h_{\mathrm{ess}}= \begin{cases}h(x, t) & \text { if } \rho_{\varepsilon} \in \mathcal{O}_{\mathrm{ess}} \\ 0 & \text { otherwise }\end{cases}
$$

We have the following lemmas.

Lemma 3.1 Let $\underline{\rho}, \bar{\rho}>0$ be two positive constants and let

$$
\mathcal{H}\left(\rho_{\varepsilon}\right) \triangleq H\left(\rho_{\varepsilon}\right)-H^{\prime}(\rho)\left(\rho_{\varepsilon}-\rho\right)-H(\rho) .
$$

Then there exists some constant $c>0$ independent of $\rho_{\varepsilon}$ such that

$$
\begin{aligned}
& \mathcal{H}\left(\rho_{\varepsilon}\right) \geq c\left|\rho_{\varepsilon}-\rho\right|^{2} \rho_{\varepsilon} \in[\underline{\rho}, \bar{\rho}], \\
& \mathcal{H}\left(\rho_{\varepsilon}\right) \geq c\left|\rho_{\varepsilon}-\rho\right| \rho_{\varepsilon}<\underline{\rho}, \\
& \mathcal{H}\left(\rho_{\varepsilon}\right) \geq c \rho_{\varepsilon} \rho_{\varepsilon}>\bar{\rho} .
\end{aligned}
$$

The proof of the lemma can be found in $[2,7]$ and is therefore omitted here for simplicity.

Lemma 3.2 Under the conditions of Lemma 3.1, there exists some positive constant $c>0$ independent of $\rho_{\varepsilon}$ such that

$$
\mathcal{H}\left(\rho_{\varepsilon}\right) \geq c\left(1+\rho_{\varepsilon}^{\gamma}\right) \quad \text { for } \rho_{\varepsilon} \in \mathcal{O}_{\text {res }}
$$

Proof By the definition of $H\left(\rho_{\varepsilon}\right)$, we have

$$
H\left(\rho_{\varepsilon}\right)=\rho_{\varepsilon} \int_{\underline{\rho}}^{\rho_{\varepsilon}} \frac{P(s)}{s^{2}} d s=\frac{1}{\gamma-1}\left(\rho_{\varepsilon}^{\gamma}-\rho_{\varepsilon} \underline{\rho}^{\gamma-1}\right) .
$$

Then

$$
\begin{aligned}
\mathcal{H}\left(\rho_{\varepsilon}\right) & =H\left(\rho_{\varepsilon}\right)-H^{\prime}(\rho)\left(\rho_{\varepsilon}-\rho\right)-H(\rho) \\
& \geq \frac{1}{\gamma-1}\left(\rho_{\varepsilon}^{\gamma}-\rho_{\varepsilon} \underline{\rho}^{\gamma-1}\right)-\left|H^{\prime}(\rho)\left(\rho_{\varepsilon}-\rho\right)\right|-|H(\rho)| \\
& \geq c \rho_{\varepsilon}^{\gamma}-c \rho_{\varepsilon}-c\left|\rho_{\varepsilon}-\rho\right|-c,
\end{aligned}
$$


that is,

$$
\mathcal{H}\left(\rho_{\varepsilon}\right)+c \rho_{\varepsilon}+c\left|\rho_{\varepsilon}-\rho\right|+c \geq c \rho_{\varepsilon}^{\gamma} .
$$

According to Lemma 5.1 in [7] there exists a constant $c>0$ such that

$$
\begin{aligned}
\mathcal{H}\left(\rho_{\varepsilon}\right) & \geq \inf _{\xi \in \partial \mathcal{O} \text { ess }}\left\{H(\xi)-H^{\prime}(\rho)(\xi-\rho)-H(\rho)\right\} \\
& =c(\underline{\rho}, \bar{\rho})>0, \quad \text { for all } \rho_{\varepsilon} \in \mathcal{O}_{\text {res }} .
\end{aligned}
$$

On the other hand, we know from Lemma 3.1 that

$$
\mathcal{H}\left(\rho_{\varepsilon}\right) \geq c\left|\rho_{\varepsilon}-\rho\right|=c\left(\rho-\rho_{\varepsilon}\right) \geq c \rho_{\varepsilon}, \quad \text { for } \rho_{\varepsilon}<\underline{\rho} \leq \frac{1}{2} \rho,
$$

which together with (3.2) gives

$$
\mathcal{H}\left(\rho_{\varepsilon}\right) \geq c\left|\rho_{\varepsilon}-\rho\right|+c \rho_{\varepsilon}+c, \quad \text { for } \rho_{\varepsilon}<\underline{\rho} \leq \frac{1}{2} \rho .
$$

Similarly to the above inequality, one has

$$
\mathcal{H}\left(\rho_{\varepsilon}\right) \geq c \rho_{\varepsilon} \geq c\left(\rho_{\varepsilon}-\rho\right)=c\left|\rho_{\varepsilon}-\rho\right|, \quad \text { for } \rho_{\varepsilon}>\bar{\rho}>\rho,
$$

then

$$
\mathcal{H}\left(\rho_{\varepsilon}\right) \geq c \rho_{\varepsilon}+c\left|\rho_{\varepsilon}-\rho\right|+c, \quad \text { for } \rho_{\varepsilon}>\bar{\rho}>\rho .
$$

In conclusion, we obtain

$$
\mathcal{H}\left(\rho_{\varepsilon}\right) \geq c\left|\rho_{\varepsilon}-\rho\right|+c \rho_{\varepsilon}+c, \quad \text { for } \rho_{\varepsilon} \in \mathcal{O}_{\text {res }},
$$

which together with (3.1) and (3.2) completes the proof.

Lemma 3.3 Let $\Omega_{\varepsilon}=(0, \varepsilon) \times(0,1)$ and $u_{\varepsilon} \in W^{1,2}\left(\Omega_{\varepsilon}\right)$ be such that $u_{\varepsilon} \cdot n=0$ on $\partial \Omega_{\varepsilon}$. Then there exists some positive constant $c$ independent of $\varepsilon$ such that

$$
c \int_{\Omega_{\varepsilon}}\left|\nabla_{x} u_{\varepsilon}\right|^{2} d x \leq \int_{\Omega_{\varepsilon}} \mathbb{S}\left(\nabla_{x} u_{\varepsilon}\right): \nabla_{x} u_{\varepsilon} d x
$$

Proof By straightforward calculation, we can get

$$
\begin{aligned}
& \int_{\Omega_{\varepsilon}}\left(\mu\left(\nabla_{x} u_{\varepsilon}+\nabla_{x}^{t} u_{\varepsilon}\right)+\lambda\left(\rho_{\varepsilon}\right) \operatorname{div}_{x} u_{\varepsilon} \mathbb{I}\right): \nabla_{x} u_{\varepsilon} d x \\
& \quad=\int_{\Omega_{\varepsilon}} \mu\left(\partial_{i} u_{\varepsilon}^{j}+\partial_{j} u_{\varepsilon}^{i}\right) \partial_{i} u_{\varepsilon}^{j}+\lambda\left(\rho_{\varepsilon}\right)\left(\operatorname{div}_{x} u_{\varepsilon} \partial_{1} u_{\varepsilon}^{1}+\operatorname{div}_{x} u_{\varepsilon} \partial_{2} u_{\varepsilon}^{2}\right) d x \\
& \quad=\int_{\Omega_{\varepsilon}}\left(\mu\left|\nabla_{x} u_{\varepsilon}\right|^{2}+\lambda\left(\rho_{\varepsilon}\right)\left|\operatorname{div}_{x} u_{\varepsilon}\right|^{2}\right) d x+\mu \int_{\Omega_{\varepsilon}} \partial_{j} u_{\varepsilon}^{i} \partial_{i} u_{\varepsilon}^{j} d x
\end{aligned}
$$


Now, we estimate the last term of the above equality. According to the boundary condition (1.2), one obtains

$$
\begin{cases}u^{1}=\partial_{1} u^{2}=0 & \text { on }\left\{x_{1}=0, \varepsilon\right\} \\ u^{2}=\partial_{2} u^{1}=0 & \text { on }\left\{x_{2}=0,1\right\}\end{cases}
$$

Thus, using (3.4) and integrating by parts on $\Omega_{\varepsilon}$, we have

$$
\begin{aligned}
& \int_{\Omega_{\varepsilon}} \partial_{j} u_{\varepsilon}^{i} \partial_{i} u_{\varepsilon}^{j} d x \\
& \quad=\int_{0}^{1} \int_{0}^{\varepsilon} \partial_{1} u_{\varepsilon}^{i} \partial_{i} u_{\varepsilon}^{1} d x_{1} d x_{2}+\int_{0}^{\varepsilon} \int_{0}^{1} \partial_{2} u_{\varepsilon}^{i} \partial_{i} u_{\varepsilon}^{2} d x_{2} d x_{1} \\
& \quad=-\int_{0}^{1} \int_{0}^{\varepsilon} u_{\varepsilon}^{i} \partial_{i} \partial_{1} u_{\varepsilon}^{1} d x_{1} d x_{2}-\int_{0}^{\varepsilon} \int_{0}^{1} u_{\varepsilon}^{i} \partial_{i} \partial_{2} u_{\varepsilon}^{2} d x_{2} d x_{1} \\
& \quad=\int_{0}^{1} \int_{0}^{\varepsilon} \partial_{i} u_{\varepsilon}^{i} \partial_{1} u_{\varepsilon}^{1} d x_{1} d x_{2}+\int_{0}^{\varepsilon} \int_{0}^{1} \partial_{i} u_{\varepsilon}^{i} \partial_{2} u_{\varepsilon}^{2} d x_{2} d x_{1} \\
& =\int_{\Omega_{\varepsilon}}\left|\operatorname{div}_{x} u_{\varepsilon}\right|^{2} d x,
\end{aligned}
$$

which together with (3.3) yields

$$
\begin{gathered}
\int_{\Omega_{\varepsilon}}\left(\mu\left(\nabla_{x} u_{\varepsilon}+\nabla_{x}^{t} u_{\varepsilon}\right)+\lambda\left(\rho_{\varepsilon}\right) \operatorname{div}_{x} u_{\varepsilon} \mathbb{I}\right): \nabla_{x} u_{\varepsilon} d x \\
=\int_{\Omega_{\varepsilon}}\left(\mu\left|\nabla_{x} u_{\varepsilon}\right|^{2}+\left(\lambda\left(\rho_{\varepsilon}\right)+\mu\right)\left|\operatorname{div}_{x} u_{\varepsilon}\right|^{2}\right) d x .
\end{gathered}
$$

By the definition of $\mathbb{S}\left(\nabla_{x} u_{\varepsilon}\right)$, we get from (3.5)

$$
\begin{aligned}
& \int_{\Omega_{\varepsilon}} \mathbb{S}\left(\nabla_{x} u_{\varepsilon}\right): \nabla_{x} u_{\varepsilon} d x \\
& \quad=\int_{\Omega_{\varepsilon}}\left(\mu\left|\nabla_{x} u_{\varepsilon}\right|^{2}+\left(\lambda\left(\rho_{\varepsilon}\right)+\mu\right)\left|\operatorname{div}_{x} u_{\varepsilon}\right|^{2}\right) d x \\
& \quad \geq c \int_{\Omega_{\varepsilon}}\left|\nabla_{x} u_{\varepsilon}\right|^{2} d x,
\end{aligned}
$$

this completes the proof.

Proof of Theorem 2.1 In order to prove Theorem 2.1, we take

$$
r=\rho(y, t), \quad U=v(y, t)=\left(\begin{array}{c}
0 \\
u(y, t)
\end{array}\right) .
$$

It follows from Remark 2.1 and (2.3) that

$$
\begin{gathered}
\mathcal{E}_{\varepsilon}\left(\left[\rho_{\varepsilon}, u_{\varepsilon}\right] \mid[\rho, v]\right)+\frac{1}{\left|I_{\varepsilon}\right|} \int_{0}^{\tau} \int_{\Omega_{\varepsilon}} \mathbb{S}\left(\nabla_{x}\left(u_{\varepsilon}-v\right)\right): \nabla_{x}\left(u_{\varepsilon}-v\right) d x d t \\
\quad \leq \mathcal{E}_{\varepsilon}\left(\left[\rho_{0, \varepsilon}, u_{0, \varepsilon}\right] \mid\left[\rho_{0}, v_{0}\right]\right)+\int_{0}^{\tau} \mathcal{F}_{\varepsilon}\left(\rho_{\varepsilon}, u_{\varepsilon}, \rho, v\right) d t,
\end{gathered}
$$


where the (scaled) relative entropy functional

$$
\begin{aligned}
\mathcal{E}_{\varepsilon}( & {\left.\left[\rho_{\varepsilon}, u_{\varepsilon}\right] \mid[\rho, v]\right) } \\
& =\frac{1}{\left|I_{\varepsilon}\right|} \int_{0}^{1} \int_{I_{\varepsilon}}\left(\frac{1}{2} \rho_{\varepsilon}\left|u_{\varepsilon}-v\right|^{2}+H\left(\rho_{\varepsilon}\right)-H^{\prime}(\rho)\left(\rho_{\varepsilon}-\rho\right)-H(\rho)\right) d x_{1} d x_{2} \\
& =\frac{1}{\left|I_{\varepsilon}\right|} \int_{0}^{1} \int_{I_{\varepsilon}}\left(\frac{1}{2} \rho_{\varepsilon}\left|u_{\varepsilon}-v\right|^{2}+\mathcal{H}\left(\rho_{\varepsilon}\right)\right)
\end{aligned}
$$

and the remainder $\mathcal{F}_{\varepsilon}$ reads

$$
\begin{aligned}
\mathcal{F}_{\varepsilon}\left(\rho_{\varepsilon}, u_{\varepsilon}, \rho, v\right)= & \frac{1}{\left|I_{\varepsilon}\right|} \int_{\Omega_{\varepsilon}} \rho_{\varepsilon}\left(v_{t}+u_{\varepsilon} \cdot \nabla_{x} v\right) \cdot\left(v-u_{\varepsilon}\right) d x \\
& +\frac{1}{\left|I_{\varepsilon}\right|} \int_{\Omega_{\varepsilon}} \rho\left(v_{t}+v \cdot \nabla_{x} v\right) \cdot\left(u_{\varepsilon}-v\right) d x \\
& +\frac{1}{\left|I_{\varepsilon}\right|} \int_{\Omega_{\varepsilon}}\left[P(\rho)-P\left(\rho_{\varepsilon}\right)+\left(\rho_{\varepsilon}-\rho\right) P^{\prime}(\rho)\right] \operatorname{div}_{x} v d x \\
& +\frac{1}{\left|I_{\varepsilon}\right|} \int_{\Omega_{\varepsilon}}\left(\rho_{\varepsilon}-\rho\right)\left(u_{\varepsilon}-v\right) \cdot \nabla_{x} H^{\prime}(\rho) d x \\
\triangleq & \sum_{i=1}^{3} J_{i} .
\end{aligned}
$$

In order to handle the integrals on the right-hand side of (3.9), we proceed in several steps as follows.

Step 1. Observe that by (2.6) and (2.7) in Theorem 2.1, we get

$$
\begin{aligned}
& \mathcal{E}_{\varepsilon}\left(\left[\rho_{0, \varepsilon}, u_{0, \varepsilon}\right] \mid\left[\rho_{0}, v_{0}\right]\right) \\
& \quad=\frac{1}{\left|I_{\varepsilon}\right|} \int_{\Omega_{\varepsilon}}\left(\frac{1}{2} \rho_{0, \varepsilon}\left|u_{0, \varepsilon}-v_{0}\right|^{2}+H\left(\rho_{0, \varepsilon}\right)-H^{\prime}\left(\rho_{0}\right)\left(\rho_{0, \varepsilon}-\rho_{0}\right)-H\left(\rho_{0}\right)\right) d x \rightarrow 0,
\end{aligned}
$$

as $\varepsilon \rightarrow 0$. From now on, we include this term in $\Gamma(\varepsilon)$ where $\Gamma(\varepsilon) \rightarrow 0$ as $\varepsilon \rightarrow 0$.

On the other hand, by Lemma 3.3, we obtain

$$
\begin{gathered}
\int_{\Omega_{\varepsilon}} \mathbb{S}\left(\nabla_{x}\left(u_{\varepsilon}-v\right)\right): \nabla_{x}\left(u_{\varepsilon}-v\right) d x \\
\quad \geq c \int_{\Omega_{\varepsilon}}\left|\nabla_{x}\left(u_{\varepsilon}-v\right)\right|^{2} d x \\
\geq c \int_{\Omega_{\varepsilon}}\left|\partial_{y}\left(u_{\varepsilon}^{2}-u\right)\right|^{2} d x,
\end{gathered}
$$

Due to the condition $\left.u_{\varepsilon} \cdot n\right|_{\partial \Omega_{\varepsilon}}=0$ and $\left.\nu \cdot n\right|_{\partial \Omega}=0$, we know that $u_{\varepsilon}^{2}=u=0$ on $\{y=0,1\}$, and then, by the Poincaré inequality, we have

$$
c \int_{\Omega_{\varepsilon}}\left|u_{\varepsilon}^{2}-u\right|^{2} d x \leq \int_{\Omega_{\varepsilon}}\left|\partial_{y}\left(u_{\varepsilon}^{2}-u\right)\right|^{2} d x
$$


Chang Journal of Inequalities and Applications

(2020) $2020: 138$

Page 11 of 16

which together with (3.11) gives

$$
\int_{\Omega_{\varepsilon}} \mathbb{S}\left(\nabla_{x}\left(u_{\varepsilon}-v\right)\right): \nabla_{x}\left(u_{\varepsilon}-v\right) d x \geq c \int_{\Omega_{\varepsilon}}\left|u_{\varepsilon}^{2}-u\right|^{2} d x .
$$

Step 2. By Eq. $(1.4)_{2}$, we have

$$
\begin{aligned}
J_{1}= & \frac{1}{\left|I_{\varepsilon}\right|} \int_{\Omega_{\varepsilon}} \rho_{\varepsilon}\left(v_{t}+u_{\varepsilon} \cdot \nabla_{x} v\right) \cdot\left(v-u_{\varepsilon}\right) d x \\
& +\frac{1}{\left|I_{\varepsilon}\right|} \int_{\Omega_{\varepsilon}} \rho\left(v_{t}+v \cdot \nabla_{x} v\right) \cdot\left(u_{\varepsilon}-v\right) d x \\
= & \frac{1}{\left|I_{\varepsilon}\right|} \int_{\Omega_{\varepsilon}}\left[\left(\rho-\rho_{\varepsilon}\right) v_{t}+\left(\rho v-\rho_{\varepsilon} u_{\varepsilon}\right) \cdot \nabla_{x} v\right] \cdot\left(u_{\varepsilon}-v\right) d x \\
= & \frac{1}{\left|I_{\varepsilon}\right|} \int_{\Omega_{\varepsilon}}\left[\left(\rho-\rho_{\varepsilon}\right) v_{t}+\left(\rho-\rho_{\varepsilon}\right) v \cdot \nabla_{x} v+\rho_{\varepsilon}\left(v-u_{\varepsilon}\right) \cdot \nabla_{x} v\right] \cdot\left(u_{\varepsilon}-v\right) d x \\
= & \frac{1}{\left|I_{\varepsilon}\right|} \int_{\Omega_{\varepsilon}}\left(\rho-\rho_{\varepsilon}\right)\left(v_{t}+v \cdot \nabla_{x} v\right) \cdot\left(u_{\varepsilon}-v\right) d x+\frac{1}{\left|I_{\varepsilon}\right|} \int_{\Omega_{\varepsilon}} \rho_{\varepsilon}\left(v-u_{\varepsilon}\right) \cdot \nabla_{x} v \cdot\left(u_{\varepsilon}-v\right) d x \\
= & \frac{1}{\left|I_{\varepsilon}\right|} \int_{\Omega_{\varepsilon}}\left(\rho-\rho_{\varepsilon}\right)\left(u_{t}+u \partial_{y} u\right)\left(u_{\varepsilon}^{2}-u\right) d x-\frac{1}{\left|I_{\varepsilon}\right|} \int_{\Omega_{\varepsilon}} \rho_{\varepsilon}\left(u_{\varepsilon}^{2}-u\right)^{2} \partial_{y} u d x \\
= & \frac{1}{\left|I_{\varepsilon}\right|} \int_{\Omega_{\varepsilon}} \frac{\rho-\rho_{\varepsilon}}{\rho}\left(u_{\varepsilon}^{2}-u\right)\left[\partial_{y}\left(v(\rho) \partial_{y} u-P(\rho)\right)\right] d x-\frac{2}{\left|I_{\varepsilon}\right|} \int_{\Omega_{\varepsilon}} \frac{1}{2} \rho_{\varepsilon}\left(u_{\varepsilon}^{2}-u\right)^{2} \partial_{y} u d x \\
\leq & c h_{1}(t) \frac{K_{1}}{\left|I_{\varepsilon}\right|}+c h_{2}(t) \mathcal{E}_{\varepsilon}\left(\left[\rho_{\varepsilon}, u_{\varepsilon}\right] \mid[\rho, v]\right),
\end{aligned}
$$

where

$$
\begin{aligned}
& h_{1}(t) \triangleq\left\|\partial_{y}\left(v(\rho) \partial_{y} u(\cdot, t)-P(\rho)(\cdot, t)\right)\right\|_{L^{\infty}(0,1)}, \\
& h_{2}(t) \triangleq\left\|\partial_{y} u(\cdot, t)\right\|_{L^{\infty}(0,1)} \\
& K_{1} \triangleq \int_{\Omega_{\varepsilon}}\left|\rho_{\varepsilon}-\rho\right|\left|u_{\varepsilon}^{2}-u\right| d x
\end{aligned}
$$

Now we estimate $h_{1}(t), h_{2}(t)$ and $K_{1}$, respectively. Firstly, we split $K_{1}$ into three parts:

$$
\begin{aligned}
& \int_{\Omega_{\varepsilon}}\left|\rho_{\varepsilon}-\rho\right|\left|u_{\varepsilon}^{2}-u\right| d x \\
& =\int_{\left\{\underline{\rho} \leq \rho_{\varepsilon} \leq \bar{\rho}\right\}}\left|\rho_{\varepsilon}-\rho\right|\left|u_{\varepsilon}^{2}-u\right| d x+\int_{\left\{\rho_{\varepsilon}<\underline{\rho}\right\}}\left|\rho_{\varepsilon}-\rho\right|\left|u_{\varepsilon}^{2}-u\right| d x \\
& \quad+\int_{\left\{\rho_{\varepsilon}>\rho\right\}}\left|\rho_{\varepsilon}-\rho\right|\left|u_{\varepsilon}^{2}-u\right| d x .
\end{aligned}
$$

Using Lemma 3.1 and Cauchy-Schwarz's inequality, we obtain

$$
\begin{aligned}
& \int_{\left\{\underline{\rho} \leq \rho_{\varepsilon} \leq \bar{\rho}\right\}}\left|\rho_{\varepsilon}-\rho\right|\left|u_{\varepsilon}^{2}-u\right| d x \\
& \quad \leq \delta \int_{\Omega_{\varepsilon}}\left|u_{\varepsilon}^{2}-u\right|^{2} d x+c(\delta) \int_{\left\{\underline{\rho} \leq \rho_{\varepsilon} \leq \bar{\rho}\right\}}\left|\rho_{\varepsilon}-\rho\right|^{2} d x
\end{aligned}
$$




$$
\begin{aligned}
& \leq \delta \int_{\Omega_{\varepsilon}}\left|u_{\varepsilon}^{2}-u\right|^{2} d x+c(\delta) \int_{\underline{\left\{\underline{\rho} \leq \rho_{\varepsilon} \leq \bar{\rho}\right\}}} \mathcal{H}\left(\rho_{\varepsilon}\right) d x \\
& \leq \delta \int_{\Omega_{\varepsilon}}\left|u_{\varepsilon}^{2}-u\right|^{2} d x+c(\delta)\left|I_{\varepsilon}\right| \mathcal{E}_{\varepsilon}\left(\left[\rho_{\varepsilon}, u_{\varepsilon}\right] \mid[\rho, v]\right)
\end{aligned}
$$

for any $\delta>0$.

Similarly to (3.15), for any $\delta>0$, one has

$$
\begin{aligned}
& \int_{\left\{\rho_{\varepsilon}<\underline{\rho}\right\}}\left|\rho_{\varepsilon}-\rho\right|\left|u_{\varepsilon}^{2}-u\right| d x \\
& \quad \leq \delta \int_{\Omega_{\varepsilon}}\left|u_{\varepsilon}^{2}-u\right|^{2} d x+c(\delta) \int_{\left\{\rho_{\varepsilon}<\underline{\rho}\right\}}\left|\rho_{\varepsilon}-\rho\right|^{2} d x \\
& \quad \leq \delta \int_{\Omega_{\varepsilon}}\left|u_{\varepsilon}^{2}-u\right|^{2} d x+c(\delta, \underline{\rho}, \bar{\rho}) \int_{\left\{\rho_{\varepsilon}<\underline{\rho}\right\}}\left|\rho_{\varepsilon}-\rho\right| d x \\
& \quad \leq \delta \int_{\Omega_{\varepsilon}}\left|u_{\varepsilon}^{2}-u\right|^{2} d x+c(\delta, \underline{\rho}, \bar{\rho}) \int_{\Omega_{\varepsilon}} \mathcal{H}\left(\rho_{\varepsilon}\right) d x \\
& \quad \leq \delta \int_{\Omega_{\varepsilon}}\left|u_{\varepsilon}^{2}-u\right|^{2} d x+c(\delta, \underline{\rho}, \bar{\rho})\left|I_{\varepsilon}\right| \mathcal{E}_{\varepsilon}\left(\left[\rho_{\varepsilon}, u_{\varepsilon}\right] \mid[\rho, v]\right) .
\end{aligned}
$$

Finally, it follows from $\rho_{\varepsilon}>\bar{\rho}$ that

$$
\left|\rho_{\varepsilon}-\rho\right|^{2}=\rho_{\varepsilon}^{2}-2 \rho_{\varepsilon} \rho+\rho^{2} \leq \rho_{\varepsilon}^{2}+\rho^{2} \leq \rho_{\varepsilon}^{2}+\bar{\rho}^{2} \leq 2 \rho_{\varepsilon}^{2},
$$

thus

$$
\begin{aligned}
& \int_{\left\{\rho_{\varepsilon}>\bar{\rho}\right\}}\left|\rho_{\varepsilon}-\rho\right|\left|u_{\varepsilon}^{2}-u\right| d x \\
& \quad \leq c \int_{\Omega_{\varepsilon}} \rho_{\varepsilon}\left|u_{\varepsilon}^{2}-u\right|^{2} d x+c \int_{\left\{\rho_{\varepsilon}>\bar{\rho}\right\}} \frac{\left|\rho_{\varepsilon}-\rho\right|^{2}}{\rho_{\varepsilon}} d x \\
& \quad \leq c \int_{\Omega_{\varepsilon}} \rho_{\varepsilon}\left|u_{\varepsilon}-v\right|^{2} d x+c \int_{\left\{\rho_{\varepsilon}>\bar{\rho}\right\}} \rho_{\varepsilon} d x \\
& \quad \leq c\left|I_{\varepsilon}\right| \mathcal{E}_{\varepsilon}\left(\left[\rho_{\varepsilon}, u_{\varepsilon}\right] \mid[\rho, v]\right) .
\end{aligned}
$$

Combining with (3.14)-(3.17), we have

$$
K_{1} \leq \delta \int_{\Omega_{\varepsilon}}\left|u_{\varepsilon}^{2}-u\right|^{2} d x+c(\delta)\left|I_{\varepsilon}\right| \mathcal{E}_{\varepsilon}\left(\left[\rho_{\varepsilon}, u_{\varepsilon}\right] \mid[\rho, v]\right)
$$

which together with (3.13), and by the regularity of $(\rho, u)$, leaves us with

$$
J_{1} \leq \delta \frac{h_{1}(t)}{\left|I_{\varepsilon}\right|} \int_{\Omega_{\varepsilon}}\left|u_{\varepsilon}^{2}-u\right|^{2} d x+c(\delta)\left[h_{1}(t)+h_{2}(t)\right] \mathcal{E}_{\varepsilon}\left(\left[\rho_{\varepsilon}, u_{\varepsilon}\right] \mid[\rho, v]\right),
$$

for any $\delta>0$. 
Step 3. We split $J_{2}$ into the residual and essential parts:

$$
\begin{aligned}
J_{2}= & \frac{1}{\left|I_{\varepsilon}\right|} \int_{\Omega_{\varepsilon}}\left[P(\rho)-P\left(\rho_{\varepsilon}\right)+\left(\rho_{\varepsilon}-\rho\right) P^{\prime}(\rho)\right] \operatorname{div}_{x} v d x \\
\leq & \left\|\partial_{y} u\right\|_{L^{\infty}(0,1)} \frac{1}{\left|I_{\varepsilon}\right|} \int_{\Omega_{\varepsilon}}\left|\left[P(\rho)-P\left(\rho_{\varepsilon}\right)+\left(\rho_{\varepsilon}-\rho\right) P^{\prime}(\rho)\right]_{\mathrm{ess}}\right| d x \\
& +\left\|\partial_{y} u\right\|_{L^{\infty}(0,1)} \frac{1}{\left|I_{\varepsilon}\right|} \int_{\Omega_{\varepsilon}}\left|\left[P(\rho)-P\left(\rho_{\varepsilon}\right)+\left(\rho_{\varepsilon}-\rho\right) P^{\prime}(\rho)\right]_{\mathrm{res}}\right| d x \\
= & \frac{h_{2}(t)}{\left|I_{\varepsilon}\right|}\left(K_{2}+K_{3}\right),
\end{aligned}
$$

where

$$
\begin{aligned}
& K_{2} \triangleq \int_{\Omega_{\varepsilon}}\left|\left[P(\rho)-P\left(\rho_{\varepsilon}\right)+\left(\rho_{\varepsilon}-\rho\right) P^{\prime}(\rho)\right]_{\mathrm{ess}}\right| d x, \\
& K_{3} \triangleq \int_{\Omega_{\varepsilon}}\left|\left[P(\rho)-P\left(\rho_{\varepsilon}\right)+\left(\rho_{\varepsilon}-\rho\right) P^{\prime}(\rho)\right]_{\mathrm{res}}\right| d x .
\end{aligned}
$$

We immediately see from Lemma 3.1 and the Taylor theorem that

$$
\begin{aligned}
K_{2} & \leq c \int_{\Omega_{\varepsilon}}\left[\left|\rho_{\varepsilon}-\rho\right|^{2}\right]_{\mathrm{ess}} d x \\
& \leq c \int_{\Omega_{\varepsilon}} \mathcal{H}\left(\rho_{\varepsilon}\right) d x \\
& \leq c\left|I_{\varepsilon}\right| \mathcal{E}_{\varepsilon}\left(\left[\rho_{\varepsilon}, u_{\varepsilon}\right] \mid[\rho, v]\right) .
\end{aligned}
$$

Next, we show that similar estimates hold also for the residual parts. Firstly, by Lemma 3.2 and Cauchy-Schwarz's inequality, for any $\alpha \in[1, \gamma]$, we have

$$
\begin{aligned}
\int_{\Omega_{\varepsilon}}\left|\left[\rho_{\varepsilon}^{\alpha}-\rho^{\alpha}\right]_{\mathrm{res}}\right| d x & \leq c \int_{\Omega_{\varepsilon}}\left|\left[\rho_{\varepsilon}\right]_{\mathrm{res}}\right|^{\alpha}+1_{\mathrm{res}} d x \\
& \leq c \int_{\Omega_{\varepsilon}}\left|\left[\rho_{\varepsilon}\right]_{\mathrm{res}}\right|^{\gamma} d x+c \int_{\Omega_{\varepsilon}} 1_{\mathrm{res}} d x \\
& \leq c \int_{\Omega_{\varepsilon}} \mathcal{H}\left(\rho_{\varepsilon}\right) d x \\
& \leq c\left|I_{\varepsilon}\right| \mathcal{E}_{\varepsilon}\left(\left[\rho_{\varepsilon}, u_{\varepsilon}\right] \mid[\rho, v]\right),
\end{aligned}
$$

thus

$$
\begin{aligned}
K_{3} & \leq c \int_{\Omega_{\varepsilon}}\left|\left[\rho_{\varepsilon}^{\gamma}-\rho^{\gamma}\right]_{\mathrm{res}}\right| d x+c \int_{\Omega_{\varepsilon}}\left|\left[\rho_{\varepsilon}-\rho\right]_{\mathrm{res}}\right| d x \\
& \leq c\left|I_{\varepsilon}\right| \mathcal{E}_{\varepsilon}\left(\left[\rho_{\varepsilon}, u_{\varepsilon}\right] \mid[\rho, \nu]\right) .
\end{aligned}
$$

Combining with (3.20)-(3.22), one has

$$
J_{2} \leq \operatorname{ch}_{2}(t) \mathcal{E}_{\varepsilon}\left(\left[\rho_{\varepsilon}, u_{\varepsilon}\right] \mid[\rho, v]\right) .
$$


Step 4. It follows from (3.18) that, for any $\delta>0$, we have

$$
\begin{aligned}
J_{3}= & \frac{1}{\left|I_{\varepsilon}\right|} \int_{\Omega_{\varepsilon}}\left(\rho_{\varepsilon}-\rho\right)\left(u_{\varepsilon}-v\right) \cdot \nabla_{x} H^{\prime}(\rho) d x \\
\leq & \frac{c}{\left|I_{\varepsilon}\right|}\left\|\partial_{y} H^{\prime}(\rho)\right\|_{L^{\infty}(0,1)} \int_{\Omega_{\varepsilon}}\left|\rho_{\varepsilon}-\rho\right|\left|u_{\varepsilon}^{2}-u\right| d x \\
= & \frac{c}{\left|I_{\varepsilon}\right|}\left\|\partial_{y} H^{\prime}(\rho)\right\|_{L^{\infty}(0,1)} K_{1} \\
\leq & \delta h_{3}(t)\left(\frac{1}{\left|I_{\varepsilon}\right|} \int_{\Omega_{\varepsilon}}\left|u_{\varepsilon}^{2}-u\right|^{2} d x\right) \\
& +c(\delta) h_{3}(t) \mathcal{E}_{\varepsilon}\left(\left[\rho_{\varepsilon}, u_{\varepsilon}\right] \mid[\rho, v]\right),
\end{aligned}
$$

where

$$
h_{3}(t) \triangleq\left\|\partial_{y} H^{\prime}(\rho)\right\|_{L^{\infty}(0,1)} .
$$

Inserting (3.10), (3.11), (3.19), (3.23) and (3.24) into (3.7) and (3.9), and taking $\delta>0$ suitable small, we have

$$
\begin{aligned}
\mathcal{E}_{\varepsilon} & \left(\left[\rho_{\varepsilon}, u_{\varepsilon}\right] \mid[\rho, v]\right)(\tau)+\frac{1}{\left|I_{\varepsilon}\right|} \int_{0}^{\tau} \int_{\Omega_{\varepsilon}}\left|u_{\varepsilon}^{2}-u\right|^{2} d x d t \\
& \leq \Gamma(\varepsilon)+c \int_{0}^{\tau}\left[h_{1}(t)+h_{2}(t)+h_{3}(t)\right] \mathcal{E}_{\varepsilon}\left(\left[\rho_{\varepsilon}, u_{\varepsilon}\right] \mid[\rho, v]\right) d t .
\end{aligned}
$$

In addition, since $(\rho, u)$ is the solution of (1.4)-(1.6), we have

$$
h_{1}(t)+h_{2}(t)+h_{3}(t) \leq c .
$$

Thus, a straightforward application of Gronwall's lemma yields

$$
\mathcal{E}_{\varepsilon}\left(\left[\rho_{\varepsilon}, u_{\varepsilon}\right] \mid[\rho, v]\right)(\tau) \rightarrow 0 \quad \text { as } \varepsilon \rightarrow 0
$$

On the other hand, By using Lemma 3.1 and Lemma 3.2, we infer that

$$
\begin{aligned}
& \left|\rho_{\varepsilon}-\rho\right|^{2} \geq c\left|\rho_{\varepsilon}-\rho\right|^{\gamma}, \quad 1<\gamma \leq 2, \text { for } \rho_{\varepsilon} \in \mathcal{O}_{\text {ess }} \\
& \left|\rho_{\varepsilon}-\rho\right|^{2}=\frac{1}{\left|\rho_{\varepsilon}-\rho\right|^{\gamma-2}}\left|\rho_{\varepsilon}-\rho\right|^{\gamma} \geq c\left|\rho_{\varepsilon}-\rho\right|^{\gamma}, \quad \gamma \geq 2, \text { for } \rho_{\varepsilon} \in \mathcal{O}_{\text {ess }},
\end{aligned}
$$

and

$$
c\left(1+\rho_{\varepsilon}^{\gamma}\right) \geq c\left|\rho_{\varepsilon}-\rho\right|^{\gamma}, \quad \gamma>1, \text { for } \rho_{\varepsilon} \in \mathcal{O}_{\text {res }}
$$


Hence, it follows from (3.8), Lemma 3.1, Lemma 3.2 and (3.26)-(3.28) that

$$
\begin{aligned}
\mathcal{E}_{\varepsilon}\left(\left[\rho_{\varepsilon}, u_{\varepsilon}\right] \mid[\rho, v]\right) & \geq \frac{c}{\left|I_{\varepsilon}\right|} \int_{\Omega_{\varepsilon}}\left(\left|\left[\rho_{\varepsilon}-\rho\right]_{\mathrm{ess}}\right|^{2}+\left|\left[1+\rho_{\varepsilon}\right]_{\mathrm{res}}\right|^{\gamma}\right) d x \\
& +\frac{c}{\left|I_{\varepsilon}\right|} \int_{\Omega_{\varepsilon}} \rho_{\varepsilon}\left|u_{\varepsilon}-v\right|^{2} d x \\
& \geq \frac{c}{\left|I_{\varepsilon}\right|} \int_{\Omega_{\varepsilon}}\left(\left|\rho_{\varepsilon}-\rho\right|^{\gamma}+\rho_{\varepsilon}\left|u_{\varepsilon}-v\right|^{2}\right) d x
\end{aligned}
$$

which together with (3.25) completes the proof of Theorem 2.1.

\section{Acknowledgements}

The author would like to thank the anonymous referee for his/her helpful comments, which improved the presentation of the paper.

Funding

Not applicable.

\section{Availability of data and materials}

Not applicable.

\section{Competing interests}

The author declares that she has no competing interests.

\section{Authors' contributions}

This entire work has been completed by the author, Dr. MZ. The author read and approved the final manuscript.

\section{Authors' information}

The author of this article is Dr. Zhang. She graduated from the School of Mathematics Science, Xiamen University, and now she works in Weifang University.

\section{Publisher's Note}

Springer Nature remains neutral with regard to jurisdictional claims in published maps and institutional affiliations.

Received: 28 December 2019 Accepted: 29 April 2020 Published online: 12 May 2020

\section{References}

1. Bella, P., Feireisl, E., Novotný, A.: Dimension reduction for compressible viscous fluid. Acta Appl. Math. 134, 111-121 (2014)

2. Březina, J., Kreml, O., Mácha, V.: Dimension reduction for the full Navier-Stokes-Fourier system. J. Math. Fluid Mech. $19,659-683(2017)$

3. Dafermos, C.M.: The second law of thermodynamics and stability. Arch. Ration. Mech. Anal. 70, 167-179 (1979)

4. Ding, S.J., Wen, H.Y., Zhu, C.J.: Global classical large solutions to 1D compressible Navier-Stokes equations with density-dependent viscosity and vacuum. J. Differ. Equ. 251, 1696-1725 (2011)

5. Ducomet, B., Caggio, M., Nečasová, Š., Pokorný, M.: The rotating Navier-Stokes-Fourier-Poisson system on thin domains. Asymptot. Anal. 109, 3-4 (2016)

6. Feireisl, E., Jin, B.J., Novotný, A.: Relative entropies, suitable weak solutions, and weak-strong uniqueness for the compressible Navier-Stokes system. J. Math. Fluid Mech. 14, 717-730 (2012)

7. Feireisl, E., Novotný, A.: Singular Limits in Thermodynamics of Viscous Fluids. Birkhäuser, Berlin (2009)

8. Feireisl, E., Novotný, A., Sun, Y.: Suitable weak solutions to the Navier-Stokes equations of compressible viscous fluids. Indiana Univ. Math. J. 60, 611-631 (2011)

9. Germain, P.: Weak-strong uniqueness for the isentropic compressible Navier-Stokes system. J. Math. Fluid Mech. 13(1), 137-146 (2011)

10. Huang, X.D., Li, J.: Existence and blowup behavior of global strong solutions to the two-dimensional barotropic compressible Navier-Stokes system with vacuum and large initial data. J. Math. Pures Appl. 106, 123-154 (2016)

11. Iftimie, D., Raugel, G., Sell, G.R.: Navier-Stokes equations in the 3D domains with Navier-boundary conditions. Indiana Univ. Math. J. 56(3), 1083-1156 (2007)

12. Kawohl, B.: Global existence of large solutions to initial boundary problems for a viscous, heat-conducting, one-dimensional real gas. J. Differ. Equ. 58, 76-103 (1985)

13. Kazhikhov, A.V., Shelukhin, V.V.: Unique global solution with respect to time of initial-boundary value problems for one-dimensional equations of a viscous gas. Prikl. Mat. Meh. 41, 282-291 (1977)

14. Lewicka, M., Müller, S.: The uniform Korn-Poincaré inequality in thin domains. Ann. Inst. Henri Poincaré, Anal. Non Linéaire 28(3), 443-469 (2011)

15. Loĭtsyanskiĭ, L.G.: Mechanics of Liquids and Gases. Nauka, Moscow (1970) (in Russian) 
16. Mellet, A., Vasseur, A.: Existence and uniqueness of global strong solutions for one-dimensional compressible Navier-Stokes equations. SIAM J. Math. Anal. 39(4), 1344-1365 (2007/08)

17. Raugel, G., Sell, G.R.: Navier-Stokes equations in thin 3D domains III. Existence of a global attractor. In: Turbulence in Fluid Flows. IMA Volumes in Mathematics and Its Applications, vol. 55, pp. 137-163. Springer, New York (1993)

18. Raugel, G., Sell, G.R.: Navier-Stokes equations in thin 3D domains I. Global attractors and global regularity of solutions. J. Am. Math. Soc. 6(3), 503-568 (1993)

19. Raugel, G., Sell, G.R.: Navier-Stokes equations in thin 3D domains II. Global regularity of spatially periodic solutions. In: Nonlinear Partial Differential Equations and Their Applications, Collège de France Seminar, vol. XI, Paris, 1989-1991. Pitman Res. Notes Math. Ser., pp. 205-247. Longman, Harlow (1994)

20. Sedov, L.I.: Continuum Mechanics, vol. 1. Nauka, Moscow (1970) (in Russian)

21. Vaigant, V.A., Kazhikhov, A.V.: On existence of global solutions to the two-dimensional Navier-Stokes equations for a compressible viscous fluid. Sib. Math. J. 36(6), 1120-1133 (1995)

22. Vodák, R.: Asymptotic analysis of steady and nonsteady Navier-Stokes equations for barotropic compressible flow. Acta Appl. Math. 110(2), 991-1009 (2010)

\section{Submit your manuscript to a SpringerOpen ${ }^{\circ}$ journal and benefit from:}

- Convenient online submission

Rigorous peer review

- Open access: articles freely available online

- High visibility within the field

- Retaining the copyright to your article

Submit your next manuscript at $\gg$ springeropen.com 